\title{
FLAME DETECTION IN VIDEO USING HIDDEN MARKOV MODELS
}

\author{
B. Uğur Töreyin, Yiğithan Dedeoğlu, A. Enis Çetin \\ Bilkent University, TR-06800 Bilkent, Ankara, Turkey
}

\begin{abstract}
This paper proposes a novel method to detect flames in video by processing the data generated by an ordinary camera monitoring a scene. In addition to ordinary motion and color clues, flame flicker process is also detected by using a hidden Markov model. Markov models representing the flame and flame colored ordinary moving objects are used to distinguish flame flicker process from motion of flame colored moving objects. Spatial color variations in flame are also evaluated by the same Markov models, as well. These clues are combined to reach a final decision. False alarms due to ordinary motion of flame colored moving objects are greatly reduced when compared to the existing video based fire detection systems.
\end{abstract}

\section{INTRODUCTION}

Conventional point smoke and fire detectors typically detect the presence of certain particles generated by smoke and fire by ionisation or photometry. An important weakness of point detectors is that they are distance limited and fail in open or large spaces. The strength of using video in fire detection is the ability to monitor large and open spaces. Current fire and flame detection algorithms are based on the use of color and motion information in video [1]. In this paper, we not only detect fire and flame colored moving regions but also analyze the motion. It is well-known that turbulent flames flicker with a frequency of around $10 \mathrm{~Hz}$ [2]. Therefore, fire detection scheme can be made more robust by detecting periodic high-frequency behavior in flame colored moving pixels compared to existing fire detection systems described in [1], [3]. In practice, flame flicker frequency is not constant and it varies in time. In fact, variations in flame pixels can be considered as random events. Therefore, a Markov model based modeling of flame flicker process produces more robust performance compared to frequency domain based methods.

If the contours of an object exhibit rapid time-varying behavior then this is an important sign of presence of flames in the scene. This time-varying behavior is directly observable in the variations of color channel values of the pixels

Email addresses: $\{$ bugur, yigithan, cetin\}@bilkent.edu.tr under consideration. Hence, the model is built as consisting of states representing relative locations of the pixels in the color space. When trained with flame pixels off-line, such a model successfully mimics the spatio-temporal characteristics of flames. The same model is also trained with nonflame pixels in order to differentiate between real flames and other flame colored ordinary moving objects. In addition, there is spatial color variation in flames. This variation can be also modeled using a Markov model. This way of modeling the problem results in less number of false alarms when compared with other proposed methods utilizing only color and ordinary motion information as in [1].

\section{FIRE AND FLAME DETECTION USING A MARKOV MODEL}

Methods of identifying flame in video include [1], [4]. The method in [4] only makes use of the color information. On the other hand, the scheme in [1] is based on detecting the fire colored regions in the current video first. If these fire colored regions move then they are marked as possible regions of fire in the scene monitored by a camera.

In video, the appearance of an object in which the contours, chrominance or luminosity oscillate similar to off-line trained flame data, constitutes a sign of the possible presence of flames. By incorporating temporal analysis around object boundaries, one can reduce the false alarms which may be due to flame colored ordinary moving objects. Turbulent flames flicker which significantly increase frequency content around $10 \mathrm{~Hz}$ [2]. In other words, a pixel especially at the edge of a flame could appear and disappear several times in one second of a video in a random manner. This characteristic behavior is very well suited to be modeled as a Markov model. Markov models are extensively used in speech recognition systems and recently they have been used in computer vision applications [5].

In [6], the shape of fire regions are represented in Fourier domain. Since, Fourier Transform does not carry any time information, FFTs have to be computed in windows of data and temporal window size is very important for detection. If it is too long then one may not get enough peaks in the FFT data. If it is too short than one may completely miss cycles and therefore no peaks can be observed in the Fourier 

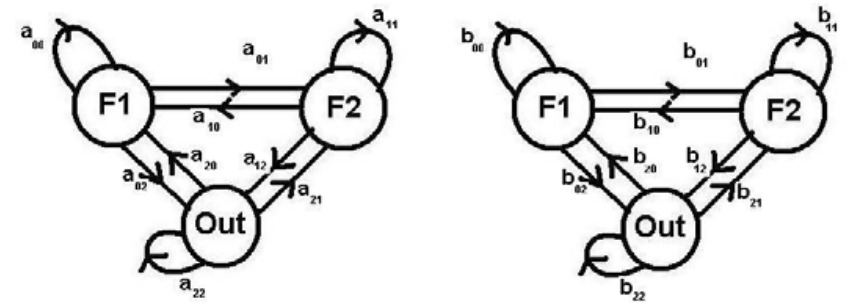

Fig. 1. Three-state Markov models for flame(left) and nonflame moving pixels.

domain. Another problem is that, one may not detect periodicity in fast growing fires because the boundary of fire region simply grows in video. However in Markov model approach, the rapid time-varying characteristic of flame boundaries, is naturally captured.

The flame regions, spatially exhibit a similar periodic behavior. Along a line passing through a flame region, the pixels' locations in the color space varies very much like those of flame pixels' observed during a period of time. In this paper, proposed Markov model is temporally trained for both flame and non-flame pixels. The transition probabilities between states for a pixel are estimated during a predetermined period of time around flame boundaries. In this way, the model not only learns the way flame boundaries flicker during a period of time, but also it tailors its parameters to mimic the spatial characteristics of flame regions. The way the model is trained as such, drastically reduces the false alarm rates. For example, a fire colored moving object will not exhibit variations in pixel values as a real flame and this will lead to a different Markov model.

\section{CHROMINANCE AND MARKOV MODELS}

Flame in color video is detected by utilizing the Markov models shown in Fig.1. Two models are trained off-line for both flame and non-flame pixels. States of the Markov models are determined according to color information. In the following sub-section, the fire color model is described. In sub-section 3.2, the Markov models are described.

\subsection{Flame Chrominance Model}

The fire and flame color model of [3] is used for defining the flame-pixels. Although there are various types of fires, fire flames especially in the initial stages of the fire exhibit a color range of red to yellow. In terms of RGB values, this fact corresponds to the following inter-relation between R, $\mathrm{G}$ and B color channels: $R>G$ and $G>B$. The combined condition for the fire region in the captured image is $R>G>B$. Besides, $\mathrm{R}$ should be more stressed than the other components, because $\mathrm{R}$ becomes the dominating color channel in an RGB image of flames. This imposes

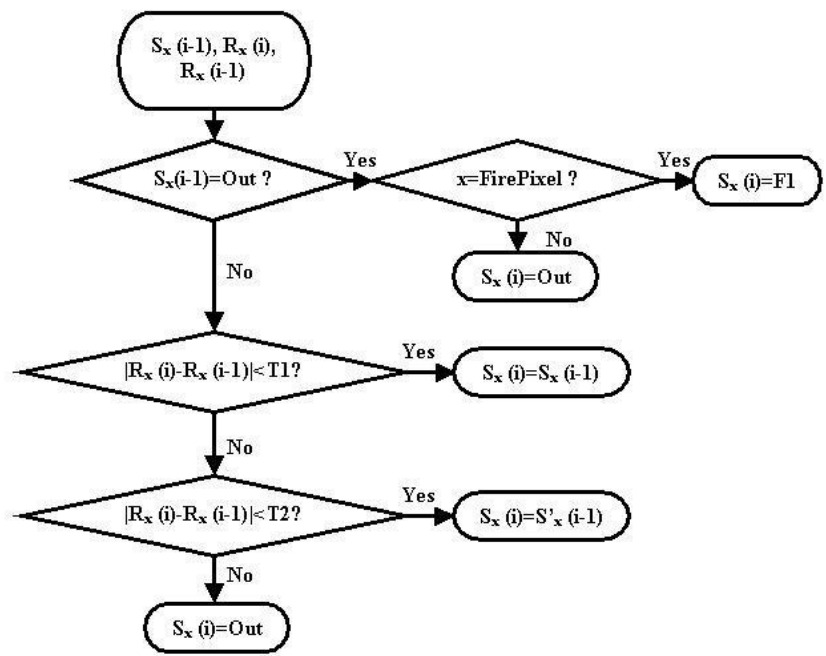

Fig. 2. State transition flow-chart of the Markov chain.

another condition for $\mathrm{R}$ as to be over some pre-determined threshold, $R_{T}$. However, lighting conditions in the background may adversely affect the saturation values of flames resulting in similar $\mathrm{R}, \mathrm{G}$ and $\mathrm{B}$ values which may cause nonflame pixels to be considered as flame colored. Therefore, saturation values of the pixels under consideration should also be over some threshold value. All of these conditions are summarized in the following composite condition:

Condition 1: $R>R_{T}$

Condition2: $R>G>B$

Condition3: $S>(255-R) * S_{T} / R_{T}$ where $S_{T}$ is the value of saturation when the value of $\mathrm{R}$ channel is $R_{T}$. If both of the three conditions are satisfied for a pixel, then that pixel is considered as a fire colored pixel. As it is known, the saturation will decrease with increasing $\mathrm{R}$ value. This is formulated in the term $(255-\mathrm{R}) * S_{T} / R_{T}$. In fire color classification, both values of $R_{T}$ and $S_{T}$ are defined according to various experimental results, and typical values range from 40 to 60 and 170 to 190 , for $S_{T}$ and $R_{T}$, respectively.

\subsection{Markov Models}

The three-state Markov model used for flame detection is presented in Fig. 1. The state $F 1$ corresponds to a pixel having a fire color defined in 3.1. The state $F 2$ also corresponds to a pixel having a fire color but the fire color range of $F 2$ is different from $F 1$. It is well known that pixel values vary inside a flame. States $F 1$ and $F 2$ represent this color variation within a flame. The state called as Out is reserved for non-fire colored pixels. Let $S_{x}(i)$ be the state of the pixel $x$ at frame $i$. The conditions for transition between states for $x$ is shown as a flow chart in Fig.2. In this diagram, $R_{x}(i)$ corresponds to the $\mathrm{R}$ channel value of the pixel $x$ at frame $i$, and $S_{x}^{\prime}(i)$ is $F 2$ if $S_{x}(i)$ is $F 1$ and vice versa. 
The thresholds should satisfy $T 1<T 2$ and they are set to 10 and 40, respectively, in our implementation. Transition between states $F 1$ and $F 2$ occurs when there is a relatively large variation in the $\mathrm{R}$ channel of the fire colored pixel. When this variation is above the larger threshold $T 2$, a transition to state $O u t$ takes place. The state of the pixel is preserved whenever a variation in the $\mathrm{R}$ channel of the fire colored pixel, which is smaller than $T 1$, is observed.

This model constitutes the basis for both temporal and spatial analysis of flame and non-flame pixels. Transition probabilities for flame and non-flame models are calculated off-line given a number of consecutive image frames. The states for flame and non-flame pixels are determined for a period of time and their corresponding transition probabilities $a_{i j}$ 's and $b_{i j}$ 's are estimated, respectively.

\section{DETECTION ALGORITHM}

Fire detection algorithm consists of four steps: (i)moving pixels or regions in the current frame of a video are determined, (ii)the colors of moving pixels are checked. If colors of pixels match to the fire colors then the hidden Markov models are used (iii)temporally and (iv)spatially to determine if fire colored pixels flicker or not.

Moving pixels and regions in the video are determined by using a background estimation method developed in [7]. In this method, a background image $B_{n+1}$ at time instant $n+1$ is recursively estimated from the image frame $I_{n}$ and the background image $B_{n}$ of the video as follows:

$$
B_{n+1}(k, l)= \begin{cases}a B_{n}(k, l)+(1-a) I_{n}(k, l) & (k, l) \text { stationary } \\ B_{n}(k, l) & (k, l) \text { moving }\end{cases}
$$

where $I_{n}(k, l)$ represent a pixel in the $n^{t h}$ video frame $I_{n}$, and $a$ is a parameter between 0 and 1. Moving pixels are determined by subtracting the current image from the background image and thresholding. A recursive threshold estimation is described in [7]. Moving regions are determined by connected component analysis. Other methods like [8] and [9] can also be used for moving pixel estimation.

The color analysis of moving pixels are carried out using the method in [3] and at moving object boundaries hidden Markov model probabilities are estimated. Each boundary pixel's state transition history of 20 frames is used to search for the model having the highest probability of generating the output sequence. The model producing the highest probability is determined. If the model representing the flame pixel has a higher probability than the other model, then spatial color analysis is performed.

Flame pixels exhibit a similar spatial variation in their chrominance or luminosity values, as shown in Fig.3. The spatial variance of flames are much larger than that of an ordinary flame-colored moving object. The absolute sum of spatial wavelet coefficients of low-high, high-low and high-

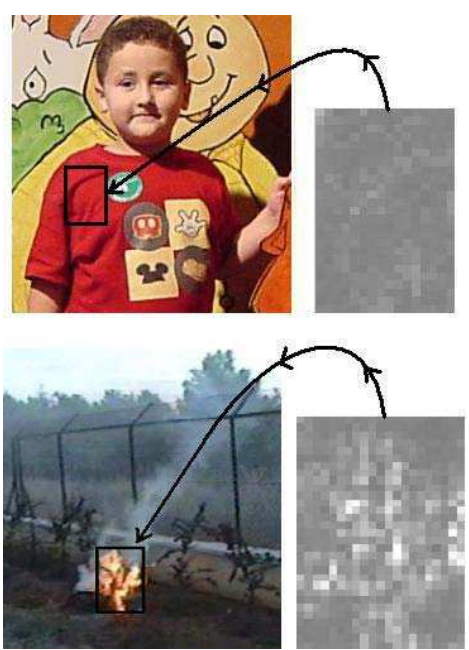

Fig. 3. Comparison of spatial variations of fire-colored regions. Flame(bottom-left) have substantially higher spatial variation(bottom-right) compared to an ordinary firecolored region.

high subimages of the regions bounded by black rectangles excerpted from a child's fire colored t-shirt and inside a fire, are shown in Fig.3, [10]. This feature of flame is also exploited by making use of the Markov models presented.

In spatial color analysis step, pixels are horizontally and vertically scanned using the same Markov models in temporal analysis. If the fire-colored model has a higher probability spatially as well, then an alarm is issued. Markov models trained using wavelet domain data can also be used to spatially analyze fire colored regions in video. This will be described in the final form of the paper.

\section{EXPERIMENTAL RESULTS}

The proposed method (Method1) is implemented in realtime in a lap-top with a Mobile AMD AthlonXP 2000+ $1.66 \mathrm{GHz}$ processor and tested for a large variety of conditions in comparison with the method utilizing only the color and temporal variation information (Method2) [1]. The computational cost of Method1 is very low, because state transition probability estimations are easily carried out with lookup tables. Method1 processes an image of size 320 by 240 at $10 \mathrm{msec}$.

The comparison results for the test sequences are presented in Table 1. Method2 is successful in determining the fire and does not recognize stationary fire-colored objects as fire, like the sun for example. However it gives false alarms when the fire-colored ordinary objects start to move. An example of this is shown in Fig.4. The fire-colored arm of the man and fire-colored parking car trigger alarm in Method2. 

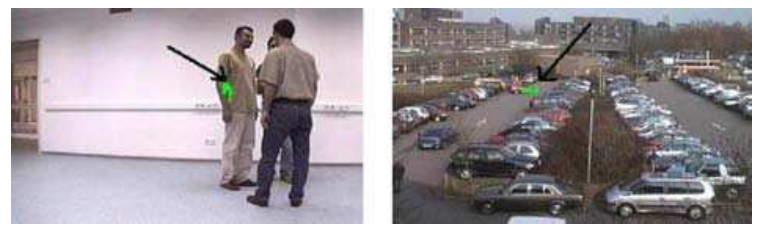

Fig. 4. False alarms issued by Method 2 on the arm of the man(left) and on the fire-colored parking car.
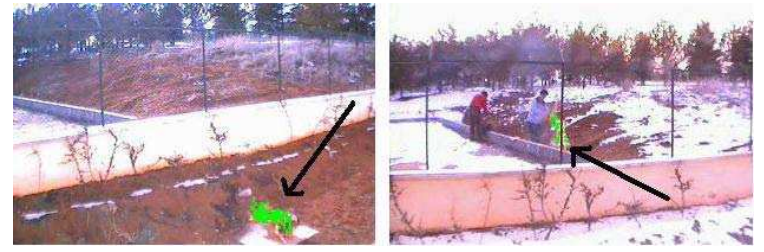

Fig. 5. Sample images from Movies 4(left) and 2. Flames are successfully detected in Movie 2 although they are partially occluded with the fence. Fire pixels are painted in bright green.

Similarly, false alarms are issued with Method 2 in Movie 7 and 9 although there are no fires taking place in these videos. Similar to the situation presented in Fig.4, these moving fire-colored objects do not cause an alarm to be raised if Method1 is used. Method1 detects fire successfully in videos covering various scenarios including partial occlusion of the flame. Sample images showing the detected regions are presented in Fig. 5.

In Movie 11 which does not contain any fires, both methods issue alarms even though Method1 drastically reduced the number of false positive frames and shots, due to its flicker process model and additional spatial analysis. In this movie, a fire colored dancing man arbitrarily waive his arms in order to fool the system. The movement of his arms just match the flame flicker process.

\section{CONCLUSION}

A robust and computationally efficient method to detect flames in color video is developed. The algorithm uses not only color and motion information, but also it detects flicker process using hidden Markov models. The same model is used for detecting spatial color variations in moving regions, as well. Methods based on only color information and ordinary movement detection may produce false alarms. Experimental results indicate that false alarms can be drastically reduced by using separate Markov models for flame and non-flame moving pixels.

The method can be used for fire detection in movies and video databases as well as real-time detection of fire. It can be incorporated with a surveillance system monitoring an
Table 1. Comparison of the proposed method (Method1) and the method based on color and temporal variation clues only (Method2).

\begin{tabular}{|c|c|c|c|c|c|}
\hline $\begin{array}{c}\text { Video } \\
\text { Sequences }\end{array}$ & $\begin{array}{l}\text { \# of Shots } \\
\text { with Fire }\end{array}$ & Method & $\begin{array}{c}\text { \# of Shots } \\
\text { detected as } \\
\text { Fire } \\
\end{array}$ & $\begin{array}{c}\text { \# of False+ } \\
\text { Frames }\end{array}$ & Description \\
\hline \multirow{2}{*}{ Movie 1} & \multirow{2}{*}{0} & Method1 & 0 & 0 & \multirow{2}{*}{$\begin{array}{l}\text { A fire-colored } \\
\text { moving truck }\end{array}$} \\
\hline & & Method2 & 5 & 46 & \\
\hline \multirow{2}{*}{ Movie 2} & \multirow{2}{*}{5} & Method1 & 5 & 0 & \multirow{2}{*}{$\begin{array}{l}\text { Fire in a } \\
\text { garden }\end{array}$} \\
\hline & & Method2 & 5 & 0 & \\
\hline \multirow{2}{*}{ Movie 3} & \multirow{2}{*}{0} & Method1 & 0 & 0 & \multirow{2}{*}{$\begin{array}{l}\text { A car leaving a } \\
\text { fire-colored } \\
\text { parking lot }\end{array}$} \\
\hline & & Method2 & 1 & 1 & \\
\hline \multirow[b]{2}{*}{ Movie 4} & \multirow[b]{2}{*}{3} & Method1 & 3 & 0 & \multirow{2}{*}{ A burning bos } \\
\hline & & Method2 & 3 & 7 & \\
\hline \multirow{2}{*}{ Movie 5} & \multirow{2}{*}{8} & Method1 & 8 & 0 & \multirow{2}{*}{$\begin{array}{l}\text { A burning pile } \\
\text { of woods }\end{array}$} \\
\hline & & Method2 & 10 & 24 & \\
\hline \multirow{2}{*}{ Movie 6} & \multirow{2}{*}{4} & Method1 & 4 & 0 & \multirow{2}{*}{$\begin{array}{l}\text { Fire behind a } \\
\text { man with a fire } \\
\text { colored shirt }\end{array}$} \\
\hline & & Method2 & 5 & 15 & \\
\hline \multirow[b]{2}{*}{ Movie 7} & \multirow[b]{2}{*}{0} & Method1 & 0 & 0 & \multirow{2}{*}{$\begin{array}{l}\text { Three men } \\
\text { walking in a } \\
\text { room }\end{array}$} \\
\hline & & Method2 & 2 & 4 & \\
\hline \multirow{2}{*}{ Movie 8} & \multirow[b]{2}{*}{2} & Method1 & 2 & 0 & \multirow{2}{*}{$\begin{array}{l}\text { Fire in a } \\
\text { fireplace }\end{array}$} \\
\hline & & Method2 & 2 & 0 & \\
\hline \multirow{2}{*}{ Movie 9} & \multirow[b]{2}{*}{0} & Method1 & 0 & 0 & \multirow{2}{*}{$\begin{array}{l}\text { A crowded } \\
\text { parking lot }\end{array}$} \\
\hline & & Method2 & 1 & 5 & \\
\hline \multirow{2}{*}{ Movie 10} & \multirow{2}{*}{0} & Method1 & 0 & 0 & \multirow{2}{*}{$\begin{array}{l}\text { Traffic on a } \\
\text { highway }\end{array}$} \\
\hline & & Method2 & 0 & 0 & \\
\hline \multirow{2}{*}{ Movie 11} & \multirow[b]{2}{*}{0} & Method1 & 3 & 8 & \multirow{2}{*}{$\begin{array}{l}\text { A fire-colored } \\
\text { dancing man }\end{array}$} \\
\hline & & Method2 & 15 & 37 & \\
\hline
\end{tabular}

indoor or an outdoor area of interest for early fire detection.

\section{REFERENCES}

[1] W. Phillips III, M. Shah, and N. V. Lobo, "Flame recognition in video," Pattern Recognition Letters, vol. 23(1-3), pp. 319327, 2002.

[2] Fastcom Technology SA, Method and Device for Detecting Fires Based on Image Analysis, Patent Coop. Treaty (PCT) Pubn.No: WO02/069292, Boulevard de Grancy 19A, CH-1006 Lausanne, Switzerland, 2002.

[3] T. Chen, P. Wu, and Y. Chiou, "An early fire-detection method based on image processing," in ICIP '04, 2004, pp. $1707-1710$.

[4] G. Healey, D. Slater, T. Lin, B. Drda, and A. D. Goedeke, "A system for real-time fire detection," in CVPR '93, 1993, pp. $15-17$.

[5] H. Bunke and T. Caelli (Eds.), HMMs Applications in Computer Vision, World Scientific, 2001.

[6] C. B. Liu and N. Ahuja, "Vision based fire detection," in ICPR '04, 2004, vol. 4.

[7] R. T. Collins, A. J. Lipton, and T. Kanade, "A system for video surveillance and monitoring," in $8^{\text {th }}$ Int. Topical Meeting on Robotics and Remote Systems. 1999, American Nuclear Society.

[8] M. Bagci, Y. Yardimci, and A. E. Cetin, "Moving object detection using adaptive subband decomposition and fractional lower order statistics in video sequences," Elsevier, Signal Processing, pp. 1941-1947, 2002.

[9] C. Stauffer and W. E. L. Grimson, "Adaptive background mixture models for real-time tracking," in CVPR '99, 1999, vol. 2.

[10] Y. Dedeoglu, B. U. Toreyin, U. Gudukbay, and A. E. Cetin, "Real-time fire and flame detection in video," in ICASSP'05. 2005, pp. 669-672, IEEE. 